\title{
On the Parameterized Complexity of Pooling Design
}

\author{
YONGXI CHENG, ${ }^{1}$ DING-ZHU DU, ${ }^{2}$ KER-I KO, ${ }^{3}$ and GUOHUI LIN ${ }^{1}$
}

\begin{abstract}
Pooling design is a very helpful tool for reducing the number of tests in DNA library screening, which is a key process to obtain high-quality DNA libraries for studying gene functions. Three basic problems in pooling design are, given an $m \times n$ binary matrix and a positive integer $d$, to decide whether the matrix is $d$-separable ( $(\bar{d}$-separable, or $d$-disjunct). The three problems are all known to be coNP-complete. Since in most applications, $d$ is a small integer compared to $n$, it is interesting to investigate whether there are efficient algorithms solving the above problems when the value of $d$ is small. In this article, we give a negative answer to the above question by studying the parameterized complexity of these three problems, with $d$ as the parameter. We show that the parameterized versions of all the three problems are co-W[2]-complete. An immediate implication of our results is that, given an $m \times n$ binary matrix and a positive integer $d$, a deterministic algorithm with running time $f(d) \times(m n){ }^{o(1)}$ (where $f$ is an arbitrary computable function) to decide whether the matrix is $d$-separable ( $\bar{d}$-separable, or $d$-disjunct) should not be expected.
\end{abstract}

Key words: disjunct matrices, DNA library screening, parameterized complexity, pooling designs, separable matrices.

\section{INTRODUCTION}

\begin{abstract}
A DNA LIBRARY CONSISTS OF THOUSANDS OF SEPARATE DNA clones. The basic task of DNA library screening is, for a collection of probes, to determine which clone from the library contains which probe. Given a probe, a clone is said to be positive if it contains the probe; otherwise, it is said to be negative. In practice, to identify all positive clones from a library, clones are often pooled together to be tested against each probe, since checking each clone-probe pair is expensive, and usually only a few clones in the library contain a given probe. An example is when Sequenced-Tagged Site markers (also called STS probes) are used (Olson et al., 1989). There are experimental tests (e.g., the Polymerase Chain Reaction) that can determine in a given pool whether or not there exists at least one clone containing a given probe.

The above application is an instance of the combinatorial group testing problem, in which we have $n$ items (each can be either positive or negative), and the number of positives is upper bounded by an integer $d$ (usually we assume that $d \ll n$ ). Suppose there exists some method that can test for any subset of items whether or not it contains at least one positive item. We say that the test outcome is positive if the test result indicates that the subset contains at least one positive item; otherwise, we say that the test outcome is
\end{abstract}

\footnotetext{
${ }^{1}$ Department of Computing Science, University of Alberta, Edmonton, Alberta, Canada.

${ }^{2}$ Department of Computer Science, University of Texas at Dallas, Richardson, Texas.

${ }^{3}$ Department of Computer Science, State University of New York at Stony Brook, Stony Brook, New York.
} 
negative. Usually the problem is to identify all positives by using the minimum number of tests. The study of group testing dates back to World War II, at first for economical mass blood testing (Dorfman, 1943). Due to its basic nature, it has found applications in many different areas. Group testing procedures can be adaptive or nonadaptive. An adaptive procedure conducts the tests one by one, and allows us to design later tests using the outcome information of all previous tests. A nonadaptive procedure specifies all tests at the beginning before knowing the outcomes of any test. The benefit is that all tests can be performed simultaneously. Between fully adaptive and nonadaptive group testing procedures, there are also two-stage procedures, which are of considerable interest (Knill, 1995; Macula, 1999; Berger et al., 2000; De Bonis et al., 2005; Eppstein et al., 2007).

\subsection{Pooling design}

Probably the most important modern applications of group testing are in the area of computational molecular biology, in which one important subject is clone library screening (Balding et al., 1995; Farach et al., 1997; Du and Hwang, 2006). In applications to molecular biology, a group testing procedure is called a pooling design, and the composition of each test is called a pool. In such applications, screening one pool at a time is far more expensive and time-consuming than screening many pools in parallel; this strongly encourages the use of nonadaptive procedures.

A nonadaptive group testing procedure can be represented as a 0-1 matrix $M=\left(m_{i j}\right)$, in which the columns are associated with the items and the rows are associated with the tests, and $m_{i j}=1$ indicates that item $j$ is contained in test $i$. The test outcomes can be represented by a $0-1$ vector, the outcome vector, where 0 indicates a negative outcome and 1 indicates a positive outcome. It is not hard to verify that if subset $S$ of columns correspond to all the positive items, then the outcome vector is equal to vector $U(S)$, the union (i.e., the componentwise Boolean sum) of all column vectors in $S$.

In order to identify all positives as long as the number of positives is no more than $d$, matrix $M$ should satisfy that for any two distinct subsets $S_{1}$ and $S_{2}$ of columns such that $\left|S_{1}\right| \leq d$ and $\left|S_{2}\right| \leq d$, $U\left(S_{1}\right) \neq U\left(S_{2}\right)$. A matrix satisfying this property is called $\bar{d}$-separable. In the definition, if we replace the condition " $\left|S_{1}\right| \leq d$ and $\left|S_{2}\right| \leq d$ " by " $\left|S_{1}\right|=\left|S_{2}\right|=d$," a matrix satisfying this property is called $d$-separable. If the matrix representing a nonadaptive pooling design is $\bar{d}$-separable (or $d$-separable), then theoretically, based on the test outcomes, we can unambiguously identify up to $d$ (or exactly $d$ ) positives from the given set. However, the actual process of determining the positives from the outcome vector, that is the decoding process, could be very time-consuming. In practice, we can adopt matrices with stronger property to make the decoding process more efficient.

For two 0-1 vectors $u$ and $v$ with the same number of components, if for any component of $u$ the value is 1 , the corresponding component of $v$ is also 1 , then we say that $u$ is covered by $v$. A $0-1$ matrix is said to be $d$-disjunct if no column is covered by the union of any $d$ other columns. The same structure is also called cover free family (Erdös et al., 1985; Ruszinkó, 1994; Füredi, 1996) in combinatorics, and superimposed code (Kautz and Singleton, 1964; D'yachkov et al., 1989, 2000) in information theory, and has been extensively studied. Obviously if a matrix is $d$-disjunct, then it is also $\bar{d}$-separable, and thus is $d$-separable. If the matrix $M$ representing a nonadaptive pooling design is $d$-disjunct and the number of positives is no more than $d$, then we have the following efficient decoding procedure with running time linear in the size of $M$ : a column $c$ corresponds to a positive item if and only if $c$ is covered by the outcome vector. $d$-Disjunct matrix is an important structure in pooling design, and a lot of work has been done on its constructions (Kautz and Singleton, 1964; Erdös et al., 1985; Hwang and Sós, 1987; Macula, 1996; D’yachkov et al., 2000; Ngo and Du, 2002; Park et al., 2003; Du et al., 2006; Fu and Hwang, 2006; Eppstein et al., 2007; Cheng and $\mathrm{Du}, 2007,2008)$.

\subsection{Main results}

Given an $m \times n$ binary matrix and a positive integer $d$, to decide whether the matrix is $d$-separable ( $\bar{d}$-separable, or $d$-disjunct) are basic problems in pooling design. They are known to be coNP-complete in classical complexity theory (Du and Ko, 1987). Thus, we shouldn't expect any polynomial time algorithm to solve any of them. However, since in most applications we have that $d \ll n$, an interesting question is whether there are efficient algorithms solving the above decision problems for small values of $d$.

In this article, by studying the parameterized complexity of the above three problems with $d$ as the parameter, we give a negative answer to the above question. More formally, we study the parameterized 
decision problems $p$-Disjunctness-Test, $p$-Separability-Test, and $p$-Separability*-Test defined as follows (where $\mathcal{N}$ denotes the set of positive integers).

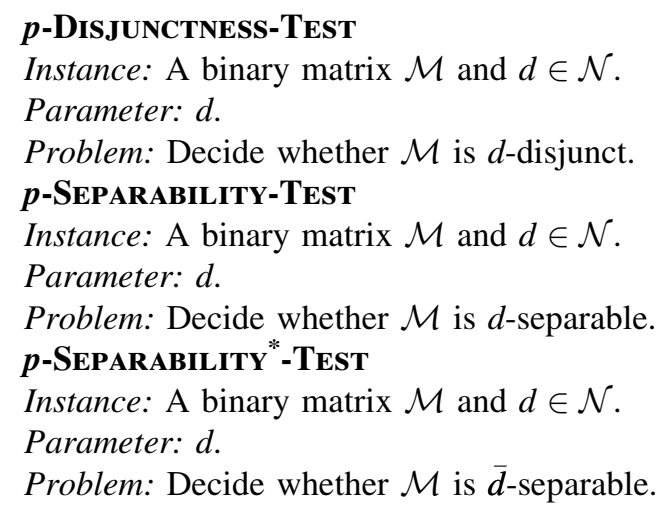

The main results of our paper are summarized in the following theorem, whose proof will be presented in Section 3.

Theorem 1.1. $p$-Disjunctness-Test, $p$-SeParability ${ }^{*}$-Test, and $p$-Separability-Test are all coW[2]-complete.

$\mathrm{W}[2]$ is the parameterized complexity class at the second level of the W-hierarchy, and co-W[2] is the class of all parameterized problems whose complements are in W[2]. They will be introduced in detail in the next section. Theorem 1.1 indicates that, given an $m \times n$ binary matrix and a positive integer $d$, a deterministic algorithm with running time $f(d) \times(m n)^{O(1)}$ (where $f$ is an arbitrary computable function) to decide whether the matrix is $d$-separable ( $\bar{d}$-separable, or $d$-disjunct) does not exist unless the class W[2] collapses to FPT (the class of all fixed-parameter tractable problems), which is commonly conjectured to be false.

\section{PRELIMINARIES}

Before proving our main results, we first briefly recall the notions of fixed-parameter tractability, relational structures, first-order logic, and the W-hierarchy of parameterized complexity classes.

\subsection{Fixed-parameter tractability}

The theory of fixed-parameter tractability (Downey and Fellows, 1999; Flum and Grohe, 2006) has received considerable attention in recent years, for both theoretical research and practical computation. In this article, we adopt the notations and conventions in Flum and Grohe (2006). Let $\Sigma$ denote a fixed finite alphabet. A parameterization of $\Sigma^{*}$ is a polynomial time computable mapping $\kappa: \Sigma^{*} \rightarrow \mathcal{N}$. A parameterized problem (over $\Sigma$ ) is a pair $(Q, \kappa)$ consisting of a set $Q \subseteq \Sigma^{*}$ and a parameterization $\kappa$ of $\Sigma^{*}$.

An algorithm $A$ with input alphabet $\Sigma$ is an fpt-algorithm with respect to $\kappa$, if for every $x \in \Sigma^{*}$ the running time of $A$ on input $x$ is at most $f(\kappa(x))|x|^{O(1)}$, for some computable function $f$. A parameterized problem $(Q, \kappa)$ is fixed-parameter tractable if there is an fpt-algorithm with respect to $\kappa$ that decides $Q$. The key point of the definition of fpt-algorithm is that the superpolynomial growth of running time is confined to the parameter $\kappa(x)$, which is usually known to be comparatively small. The class of all fixed-parameter tractable problems is denoted by FPT.

Many NP-hard problems such as the Vertex Cover problem (Chen et al., 2001) and the ML TypeChecking problem (Lichtenstein and Pnueli, 1985) have been shown to be fixed-parameter tractable. On the other hand, there is strong theoretical evidence that certain well-known parameterized problems, for instance, the Independent Set problem and the Dominating Set problem, are not fixed-parameter tractable (Downey and Fellows, 1999). This evidence is provided, similar to the theory of NP-completeness, via a completeness theory based on the following notion of reductions: Let $(Q, \kappa)$ and $\left(Q^{\prime}, \kappa^{\prime}\right)$ be 
parameterized problems over alphabets $\Sigma$ and $\Sigma^{\prime}$, respectively. An fpt-reduction from $(Q, \kappa)$ to $\left(Q^{\prime}, \kappa^{\prime}\right)$ is a mapping $R: \Sigma^{*} \rightarrow\left(\Sigma^{\prime}\right)^{*}$ such that:

1. For all $x \in \Sigma^{*}, x \in Q$ if and only if $R(x) \in Q^{\prime}$.

2. $R$ is computable by an fpt-algorithm (with respective to $\kappa$ ). That is, there is a computable function $f$ such that $R(x)$ is computable in time $f(\kappa(x))|x|^{O(1)}$.

3. There is a computable function $g: \mathcal{N} \rightarrow \mathcal{N}$ such that $\kappa^{\prime}(R(x)) \leq g(\kappa(x))$, for all $x \in \Sigma^{*}$.

In the above definition, the last requirement is to ensure that class FPT is closed under fpt-reductions, that is, if a parameterized problem $(Q, \kappa)$ is reducible to another parameterized problem $\left(Q^{\prime}, \kappa^{\prime}\right)$ and $\left(Q^{\prime}, \kappa^{\prime}\right) \in \mathrm{FPT}$, then $(Q, \kappa) \in \mathrm{FPT}$.

\subsection{Relational structures}

In later discussions, we adopt the conventions in descriptive complexity theory, in which instances of decision problems are viewed as structures of some vocabulary instead of languages over some finite alphabet.

A (relational) vocabulary $\tau$ is a set of relation symbols. Each relation symbol $R \in \tau$ has an arity $\operatorname{arity}(R) \geq 1$. A structure $\mathcal{A}$ of vocabulary $\tau$ consists of a set $A$ called the universe and an interpretation $R^{\mathcal{A}} \subseteq A^{\text {arity }(R)}$ of each relation symbol $R \in \tau$. For a tuple $\bar{a} \in A^{\text {arity }(R)}$, we write $R^{\mathcal{A}} \bar{a}$ (or $\bar{a} \in R^{\mathcal{A}}$ ) to denote that $\bar{a}$ belongs to the relation $R^{\mathcal{A}}$. In this article, we only consider nonempty finite vocabularies and structures with a finite universe.

Recall that a hypergraph is a pair $H=(V, E)$ consisting of a set $V$ of vertices and a set $E$ of hyperedges. Each hyperedge is a subset of $V$. Graphs are hypergraphs with hyperedges of cardinality two. The following example illustrates how to represent a hypergraph using a relational structure.

Example 2.1. Let $\tau_{H G}$ be the vocabulary consisting of the unary relation symbols $V E R T$ and $E D G E$ and the binary relation symbol $I$. A hypergraph $H=(V, E)$ can be represented by a relational structure $\mathcal{H}$ of vocabulary $\tau_{H G}$ as follows:

- The universe of $\mathcal{H}$ is $V \cup E$.

- $V E R T^{\mathcal{H}}:=V$ and $E D G E^{\mathcal{H}}:=E$.

- $I^{\mathcal{H}}:=\{(v, e): v \in V, e \in E$, and $v \in e\}$ is the incidence relation.

\subsection{First-order logic}

We briefly recall the syntax of first-order logic. Let $\tau$ be a vocabulary. Atomic first-order formulas of vocabulary $\tau$ are of the form $x=y$ or $R x_{1} \ldots x_{\ell}$, where $R \in \tau$ is $\ell$-ary (i.e., has arity $\ell$ ) and $x, y, x_{1}, \ldots, x_{\ell}$ are variables. First-order formulas of vocabulary $\tau$ are built from atomic formulas using Boolean connectives $\wedge$ (and), $\vee$ (or), $\neg$ (negation), together with the existential and universal quantifiers $\exists$ and $\forall$. The connectives $\rightarrow$ (implication) and $\leftrightarrow$ (equivalence) are not part of the language defining first-order formulas, but we use them as abbreviations: $\varphi \rightarrow \psi$ stands for $\neg \varphi \vee \psi$, and $\varphi \leftrightarrow \psi$ stands for $(\varphi \rightarrow \psi) \wedge(\psi \rightarrow \varphi)$.

For a variable $x$, we call $x$ a free variable of $\varphi$ if $x$ occurs in $\varphi$ but is not in the scope of a quantifier binding $x$. We write $\varphi\left(x_{1}, \ldots, x_{\ell}\right)$ to indicate that all free variables of $\varphi$ belong to set $\left\{x_{1}, \ldots, x_{\ell}\right\}$. A formula without free variables is called a sentence. Let both $\Sigma_{0}$ and $\Pi_{0}$ denote the class of quantifier-free first-order formulas. For $t \geq 0$, let $\Sigma_{t+1}$ be the class of all formulas $\left(\exists x_{1} \ldots \exists x_{\ell}\right) \varphi$, where $\varphi \in \Pi_{t}$, and let $\Pi_{t+1}$ be the class of all formulas $\left(\forall x_{1} \ldots \forall x_{\ell}\right) \varphi$, where $\varphi \in \Sigma_{t}$.

For formulas of second-order logic, in addition to the individual variables, they may also contain relation variables, each of the relation variables has a prescribed arity. We use lowercase letters (e.g., $x, y, z)$ to denote individual variables and uppercase letters (e.g., $X, Y, Z)$ to denote relation variables. As in Flum and Grohe (2006), for convenience we allow free relation variables to be in first-order formulas, since the crucial difference between first-order and second-order logic is not that second-order formulas can have relation variables, but that second-order formulas can quantify over relations. Therefore, in this paper the syntax of first-order logic is enhanced by including new atomic formulas of the form $X x_{1} \ldots x_{\ell}$, where $X$ is an $\ell$-ary relation variable. The meaning of formula $X x_{1} \ldots x_{\ell}$ is: The tuple of elements interpreting $\left(x_{1}, \ldots, x_{\ell}\right)$ is contained in the relation interpreting the relation variable $X$. We also extend classes such as $\Sigma_{t}$ and $\Pi_{t}$ to include formulas with free relation variables. It is worth emphasizing again that in first-order logic we do not allow quantification over relation variables. 


\subsection{W-hierarchy}

We give a brief introduction to the W-hierarchy of parameterized complexity classes, which plays a central role in the theory of parameterized intractability. Roughly speaking, the W-hierarchy classifies problems according to the syntactic form of their definitions, and the definitions are formalized using languages of mathematical logic. The W-hierarchy can be defined in several different ways, we adopt the following definition based on the weighted Fagin-defined problems.

Let $\varphi(X)$ be a first-order formula with a free relation variable $X$ of arity $s$. Define $p$ - $\mathrm{WD}_{\varphi}$ to be the following parameterized decision problem.

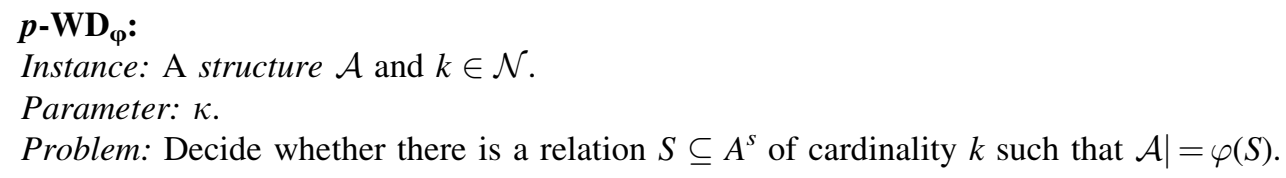

Here, $\mathcal{A} \mid=\varphi(S)$ stands for that structure $\mathcal{A}$ satisfies sentence $\varphi(S)$ (or, $\mathcal{A}$ is a model of $\varphi(S)$ ), and $S$ is called a solution for $\varphi$ in structure $\mathcal{A}$. The readers are referred to Section 4.2 of Flum and Grohe (2006) for more detailed introduction to the semantics of first-order formulas.

For a class $\Phi$ of first-order formulas, let $p-\mathrm{WD}-\Phi$ be the class of all parameterized problems $p-\mathrm{WD}_{\varphi}$ with $\varphi \in \Phi$. For $t \geq 1$, define $\mathrm{W}[t]:=\left[p-\mathrm{WD}-\Pi_{t}\right]^{f p t}$, which is the class of all parameterized problems that are fpt-reducible to some problems in $p$-WD- $\Pi_{t}$. The classes $\mathrm{W}[t]$, for $t \geq 1$, form the $\mathrm{W}$-hierarchy. Thus, the levels of W-hierarchy essentially correspond to the number of alternations between universal and existential quantifiers in the definitions of their complete problems. Problems hard for W[1] or higher class are assumed not to be fixed-parameter tractable. For instance, the InDEPENDENT SET problem is W[1]-complete and the Dominating Set problem is W[2]-complete.

For a parameterized problem $(Q, \kappa)$ over the alphabet $\Sigma$, let $(Q, \kappa)^{c}$ denote its complement, that is the parameterized problem $\left(\Sigma^{*} \backslash Q, \kappa\right)$. Let $C$ be a parameterized complexity class. Then co- $C$ is defined to be the class of all parameterized problems $(Q, \kappa)$ such that $(Q, \kappa)^{c} \in C$. Clearly, FPT $=$ co-FPT. From the definition of fpt-reductions, it is easy to see that if class $C$ is closed under fpt-reductions, so is co- $C$. In particular, each class $\mathrm{W}[t], t \geq 1$, gives rise to a new parameterized complexity class co-W[t]. Also, it is easy to prove that if $(Q, \kappa)$ is complete in parameterized complexity class $C$ under fpt-reductions, then $(Q, \kappa)^{c}$ is complete in class co- $C$ under fpt-reductions.

\section{PROOF OF THEOREM 1.1}

We devote this section to the proof of Theorem 1.1. For a binary matrix $M$, let $R_{M}$ be the set consisting of all rows in $M$ and let $C_{M}$ be the set consisting of all columns in $M$.

Relational structure for a binary matrix. Let $\tau_{B M}$ be the vocabulary consisting of the unary relation symbols ROW and COLUMN and the binary relation symbol $I$. Then, the binary matrix $M$ can be represented by a structure $\mathcal{M}$ of vocabulary $\tau_{B M}$, where the universe of $\mathcal{M}$ is $R_{M} \cup C_{M}$, and the interpretations for the relation symbols in $\tau_{B M}$ are as follows:

- $R O W^{\mathcal{M}}:=R_{M}$.

- $\operatorname{COLUMN}^{\mathcal{M}}:=C_{M}$.

- $I^{\mathcal{M}}:=\left\{(r, c): r \in R_{M}, c \in C_{M}\right.$, and $\left.M(r, c)=1\right\}$, which is the incidence relation.

The proof of Theorem 1.1 is partitioned into the following six lemmas.

Lemma 3.1. $p$-DisJunCtNess-Test $\in$ co-W[2].

Proof. We consider the following complement problem of $p$-Disjunctness-Test.

\section{$p$-NONDisJunCTNESS-TeST}

Instance: A binary matrix $\mathcal{M}$ and $d \in \mathcal{N}$.

Parameter: $d$.

Problem: Decide whether $\mathcal{M}$ is NOT $d$-disjunct. 
We will define a $\Pi_{2}$ formula nondisj $(X)$, with a free relation variable $X$ of arity 2, and show that $p$-NondisJunCtNess-Test is equal to $p$ - $\mathrm{WD}_{\text {nondisj(X) }}$ (see Section 2.4 for the definition of problem $p$ - $\mathrm{WD}_{\varphi}$ ). This implies that $p$-NondisJunctNess-Test is in $p$-WD- $\Pi_{2}$, therefore is in $\mathrm{W}[2]$.

A binary matrix is not $d$-disjunct if and only if there is a set $D$ of $d$ columns and another column $c \notin D$ such that $U(D)$ covers $c$. Our idea is assuming that the solution $S$ to $X$ is of the form $\left\{\left(c_{i}, c\right): c_{i} \in D\right\}$. Therefore, $X$ should be a binary relation variable and the solution $S$ to $X$ should have cardinality $d$.

Define $\chi_{1}:=\forall c_{1} \forall c_{2}\left(X c_{1} c_{2} \rightarrow\left(C O L U M N c_{1} \wedge C O L U M N c_{2} \wedge\left(c_{1} \neq c_{2}\right)\right)\right)$, and define $\chi_{2}:=\forall c_{3} \forall c_{4}$ $\forall c_{5} \forall c_{6}\left(\left(X c_{3} c_{4} \wedge X c_{5} c_{6}\right) \rightarrow\left(c_{4}=c_{6}\right)\right)$. Here $\chi_{1}$ and $\chi_{2}$ are to guarantee that the solution $S$ to $X$ of cardinality $d$ has the form $\left\{\left(c_{1}, c\right), \ldots,\left(c_{d}, c\right)\right\}$, where $c \in C_{M}, c_{i} \in C_{M}$, and $c_{i} \neq c$, for $1 \leq i \leq d$. Define nondisj' $(X):=\forall r \exists c_{7} \exists c_{8}\left(R O W r \rightarrow\left(X c_{7} c_{8} \wedge\left(I r c_{8} \rightarrow I r c_{7}\right)\right)\right)$. nondisj'(X) is to guarantee that, the solution $S$ to $X$ satisfies that the union of columns in $\left\{c_{i}:\left(c_{i}, c\right) \in S\right\}$ covers $c$ (so that $\mathcal{M}$ is not $d$-disjunct). Finally, define $\operatorname{nondisj}(X):=\chi_{1} \wedge \chi_{2} \wedge$ nondisj' $(X)$, which is equivalent to a $\Pi_{2}$ formula.

From the above definition, clearly if there exists a relation $S \subseteq C_{M}^{2}$ of cardinality $d$ such that $\mathcal{M} \mid=$ nondisj $(S)$, then $\mathcal{M}$ is not $d$-disjunct. On the other hand, if $\mathcal{M}$ is not $d$-disjunct, then there exist a subset $D$ of $d$ columns $c_{1}, \ldots, c_{d}$ and another column $c \notin D$ such that $c$ is covered by $U(D)$. It is not hard to verify that the relation $S=\left\{\left(c_{1}, c\right), \ldots,\left(c_{d}, c\right)\right\}$ satisfies $\mathcal{M} \mid=n o n \operatorname{disj}(S)$. Therefore, $\mathcal{M}$ is not $d$-disjunct if and only if there exists a relation $S$ of cardinality $d$ such that $\mathcal{M} \mid=\operatorname{nondisj}(S)$. That is, $p$-NondisJunctnessTest is $p$ - $\mathrm{WD}_{\text {nondisj(X) }}$. Thus, $p$-NondisJunctness-Test $\in \mathrm{W}[2]$, and so $p$-DisJunctness-Test $\in$ co$\mathrm{W}[2]$.

Lemma 3.2. $p$-Separability-Test $\in$ co-W[2].

Proof. We consider the following complement problem of $p$-Separability-Test.

\section{$p$-NONSEPARABILITY-TEST}

Instance: A binary matrix $\mathcal{M}$ and $d \in \mathcal{N}$.

Parameter: $d$.

Problem: Decide whether $\mathcal{M}$ is NOT $d$-separabe.

We will define a formula nonsep $(Y)$, with a free relation variable $Y$ of arity 2 , and show that $p$-NONSEPARABILITY-TEST is equal to $p$ - $\mathrm{WD}_{\text {nonse }(Y)}$.

A binary matrix is not $d$-separable if and only if there exist two distinct subsets $D_{1}$ and $D_{2}$ each contains $d$ columns such that $U\left(D_{1}\right)=U\left(D_{2}\right)$. Assume that $D_{1}=\left\{c_{11}, c_{12}, \ldots, c_{1 d}\right\}$ and $D_{2}=\left\{c_{21}, c_{22}, \ldots, c_{2 d}\right\}$. The idea is to assume that the solution $S$ to $Y$ is of the form $\left\{\left(c_{11}, c_{21}\right),\left(c_{12}, c_{22}\right), \ldots,\left(c_{1 d}, c_{2 d}\right)\right\}$, and so $Y$ should be a binary relation variable and the solution $S$ to $Y$ should have cardinality $d$. We define the formula nonsep $(Y)$ satisfying that, there exists a relation $S \subseteq C_{M}^{2}$ of cardinality $d$ such that $\mathcal{M} \mid=\operatorname{nonsep}(S)$ if and only if $\mathcal{M}$ is not $d$-separable.

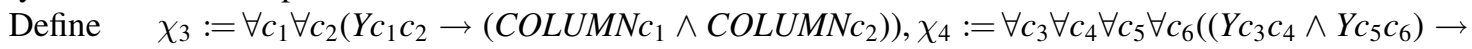
$\left.\left(\left(c_{3}=c_{5}\right) \leftrightarrow\left(c_{4}=c_{6}\right)\right)\right)$, and $\chi_{5}:=\exists c_{7} \exists c_{8} \forall c_{9}\left(\left(Y c_{7} c_{8} \wedge \neg Y c_{9} c_{7}\right)\right)$. Here $\chi_{3}$ is to guarantee that the relation variable $Y \subseteq C_{M}^{2} ; \chi_{4}$ is to build a bijection between the first component of elements in $S$ and the second component of elements in $S$, which guarantees that the two subsets $\left\{c_{1 i}: \exists\right.$ cs.t. $\left.\left(c_{1 i}, c\right) \in S\right\}$ and $\left\{c_{2 j}: \exists c s . t .\left(c, c_{2 j}\right) \in S\right\}$ (which intend to be $D_{1}$ and $D_{2}$ respectively) have the same cardinality; $\chi_{5}$ is to guarantee that the two subsets $\left\{c_{1 i}: \exists c s . t .\left(c_{1 i}, c\right) \in S\right\}$ and $\left\{c_{2 j}: \exists c s . t .\left(c, c_{2 j}\right) \in S\right\}$ are distinct from each other. Define $\operatorname{nonsep}^{\prime}(Y):=\forall r\left(R O W r \rightarrow\left(\left(\exists c_{10} \exists c_{11} Y c_{10} c_{11} \wedge \operatorname{Irc}_{10}\right) \leftrightarrow\left(\exists c_{12} \exists c_{13} Y c_{12} c_{13} \wedge \operatorname{Irc}_{13}\right)\right)\right)$, which is to guarantee that, the solution $S$ to $Y$ satisfies that the union of columns in $\left\{c_{1 i}: \exists c s . t .\left(c_{1 i}, c\right) \in S\right\}$ is equal to the union of columns in $\left\{c_{2 j}: \exists\right.$ cs.t. $\left.\left(c, c_{2 j}\right) \in S\right\}$. From basic logic computation it is not hard to verify that $\operatorname{nonsep}^{\prime}(Y)$ is a $\Pi_{2}$ formula with free relation variable $Y$. Finally, define $\operatorname{nonsep}(Y):=\chi_{3} \wedge$ $\chi_{4} \wedge \chi_{5} \wedge \operatorname{nonsep}^{\prime}(Y)$.

From the above definition of $n \operatorname{nonsep}(X)$, if a relation $S \subseteq C_{M}^{2}$ of cardinality $d$ satisfies that $\mathcal{M} \mid=$ nonsep $(S)$, then the two subsets $\left\{c_{1 i}: \exists c s . t .\left(c_{1 i}, c\right) \in S\right\}$ and $\left\{c_{2 j}: \exists c s . t .\left(c, c_{2 j}\right) \in S\right\}$ both contain $d$ columns of $\mathcal{M}$ and are distinct from each other, moreover their unions are identical. This implies that $\mathcal{M}$ is not $d$-separable. On the other hand, if $\mathcal{M}$ is not $d$-separable, then there exist two distinct subsets $D_{1}$ and $D_{2}$ each contains $d$ columns such that $U\left(D_{1}\right)=U\left(D_{2}\right)$. Assume that $D_{1}=\left\{c_{11}, \ldots, c_{1 d}\right\}$, and $D_{2}=\left\{c_{21}, \ldots, c_{2 d}\right\}$. It is not hard to verify that the relation $S=\left\{\left(c_{11}, c_{21}\right),\left(c_{12}, c_{22}\right), \ldots,\left(c_{1 d}, c_{2 d}\right)\right\}$ satisfies $\mathcal{M} \mid=\operatorname{nonsep}(S)$. 
From above, there exists a relation $S \subseteq C_{M}^{2}$ of cardinality $d$ such that $\mathcal{M} \mid=n o n \operatorname{sep}(S)$ if and only if $\mathcal{M}$ is not $d$-separable, therefore $p$-NonseParabiLITY-TeST is $p$-WD nonsep $(Y) \cdot \chi_{3}$ and $\chi_{4}$ are $\Pi_{1}$ formulas, $\chi_{5}$ is a $\Sigma_{2}$ formula, $\operatorname{nonsep}^{\prime}(Y)$ is a $\Pi_{2}$ formula, therefore $\operatorname{nonsep}(Y)=\chi_{3} \wedge \chi_{4} \wedge \chi_{5} \wedge \operatorname{nonsep}^{\prime}(Y)$ is equivalent to a $\Sigma_{3}$ formula, which implies that $p$-NonseParabiLity-TeST is in $p$-WD- $\Sigma_{3}$. Here we apply the fact that $p-W D-\Sigma_{3} \subseteq p$-WD- $\Pi_{2}{ }^{1}{ }^{1}$ Thus, $p$-Nonseparability-Test $\in \mathrm{W}[2]$, and so $p$-SeparabilityTEST $\in$ Co-W[2].

Lemma 3.3. $p$-Separability ${ }^{*}$-Test $\in$ co-W[2].

Proof. Since $\mathrm{W}[2]$ is closed under fpt-reductions, so is co-W[2]. We will show that $p$-Separability ${ }^{*}$ TeSt is fpt-reducible to $p$-SEParability-Test. Since the latter is in co-W[2] (lemma 3.2), this implies that $p$-Separability ${ }^{*}$-Test $\in$ co-W[2]. The reduction can be obtained immediately from the following fact (lemma 2.1.6 in Du and Hwang [2006]): A binary matrix $M^{\prime}$ containing a zero column is $d$-separable if and only if the matrix $M$ obtained by removing this zero column from $M^{\prime}$ is $\bar{d}$-separable.

Let $(M, d)$ be an instance of $p$-SEPARABILITY*-TEST, where $M$ is a binary matrix and the parameter $d$ is a positive integer. We map $(M, d)$ to $\left(M^{\prime}, d\right)$, where $M^{\prime}$ is obtained by adding a zero column to $M$. From the above lemma, $(M, d) \in p$-Separability*-Test if and only if $\left(M^{\prime}, d\right) \in p$-Separability-Test. It is easy to see that this is an fpt-reduction from $p$-SEParability*-Test to $p$-SeParabiLity-TeST.

Lemma 3.4. $p$-DisJunCtNESS-TeST is co-W[2]-complete.

Proof. A hitting set in a hypergraph $\mathcal{H}=(V, E)$ is a set $T$ of vertices that intersects each hyperedge, that is $T \cap e \neq \emptyset$ for all $e \in E$. The classical HitTING-SET problem is to find a hitting set of a given cardinality $k$ in a given hypergraph $\mathcal{H}$, which is known to be NP-complete. The following parameterized hitting set problem is W[2]-complete (see, Theorem 7.14 in Flum and Grohe [2006]).

\section{p-HitTING-SeT}

Instance: A hypergraph $\mathcal{H}$ and $k \in \mathcal{N}$.

Parameter: $k$.

Problem: Decide whether $\mathcal{H}$ has a hitting set of $k$ vertices.

We give an ftp-reduction from $p$-HitTING-SET to $p$-NondisJUnCTNESS-TeSt, based on an idea similar to that in $\mathrm{Du}$ and Ko (1987). Let $(\mathcal{H}, k)$ with $\mathcal{H}=(V, E)$ be an instance of $p$-Hitting-Set, where $V=\{1, \ldots, n\}, E=\left\{e_{1}, \ldots, e_{m}\right\}$, and each $e_{i}, 1 \leq i \leq m$, is a subset of $V$. Define $d=k$, and define an $(n+m) \times(n+1)$ binary matrix $M$ with rows $R_{i}$ as follows (here we represent each row as a subset of the set of all columns $\{1,2, \ldots, n+1\}$, in the most natural way).

$$
\begin{array}{rlrl}
R_{i} & =\{i\}, & & i=1, \ldots, n ; \\
R_{n+j} & =e_{j} \cup\{n+1\}, & j=1, \ldots, m .
\end{array}
$$

First, assume that $\mathcal{H}$ has a hitting set $T \subseteq V$ of size $k$. Consider the subset $S_{1}=T$ of columns of $M$. Since $T$ is a hitting set of $\mathcal{H}, U\left(S_{1}\right)$ covers column $n+1$. Notice that $\left|S_{1}\right|=d$ and column $n+1$ is not in $S_{1}, M$ is not $d$-disjunct.

Conversely, assume that $M$ is not $d$-disjunct. Then, there exist a subset $S_{1}$ of $d$ columns in $\{1,2, \ldots, n+1\}$ and another column $c \notin S_{1}$ such that $U\left(S_{1}\right)$ covers $c$. From the way we define the first $n$ rows of matrix $M, c$ can only be column $n+1$. Thus, column $n+1$ is not in $S_{1}$. Set $T=S_{1}$, then $|T|=k$ and $T$ is a subset of $V$. Since $U\left(S_{1}\right)$ covers column $n+1$, it is easy to see that $T$ is a hitting set of $\mathcal{H}$.

It is not hard to verify that the above is an fpt-reduction. Therefore, $p$-NONDISJUNCTNESS-TEST is W[2]complete, and so $p$-DisJunCTNESS-TEST is co-W[2]-complete.

Lemma 3.5. $p$-SeparabiLITY*-Test is co-W[2]-complete.

\footnotetext{
${ }^{1}$ More generally, $p-W D-\Sigma_{t+1} \subseteq p-W D-\Pi_{t}$, for $t \geq 1$. The main idea to prove this conclusion is not complicated; we refer the reader to Proposition 5.4 in Flum and Grohe (2006) for the proof.
} 
Proof. We give an ftp-reduction from $p$-Hitting-Set to $p$-Nonseparability*-Test. For an instance $(\mathcal{H}, k)$ of $p$-Hitting-Set, define matrix $M$ in the same way as in the proof of lemma 3.4 , and define $d=k+1$. Next we show the correctness of this reduction.

First, assume that $\mathcal{H}$ has a hitting set $T \subseteq V$ of size $k$. Consider the following two subsets of columns in $M: S_{1}=T$ and $S_{2}=T \cup\{n+1\}$. Then, for $1 \leq i \leq n$, it is obvious that $U\left(S_{1}\right)_{i}=U\left(S_{2}\right)_{i}$; for $n i \leq n+m$, since $T$ is a hitting set of $\mathcal{H}, U\left(S_{1}\right)_{i}=1=U\left(S_{2}\right)_{i}$. Notice that $\left|S_{1}\right|,\left|S_{2}\right| \leq d$ and $S_{1} \neq S_{2}, M$ is not $\bar{d}$-separable.

Conversely, assume that $M$ is not $\bar{d}$-separable. Then, there exist two subsets $S_{1}$ and $S_{2}$ of columns in $\{1,2, \ldots, n+1\}$ such that $\left|S_{1}\right|,\left|S_{2}\right| \leq d, S_{1} \neq S_{2}$, and $U\left(S_{1}\right)=U\left(S_{2}\right)$. Since $U\left(S_{1}\right)_{i}=U\left(S_{2}\right)_{i}$ for $1 \leq i \leq n$, we have that $S_{1} \cap\{1, \ldots, n\}=S_{2} \cap\{1, \ldots, n\}$. To have $S_{1} \neq S_{2}$, column $n+1$ must belong to exactly one of $S_{1}$ and $S_{2}$. Without loss of generality, assume that $n+1 \notin S_{1}$, and $n+1 \in S_{2}$. Set $T=S_{1}$, then $|T|=\left|S_{1}\right|=\left|S_{2}\right|-1 \leq d-1=k$, and $T$ is a subset of $V$. From $U\left(S_{1}\right)_{i}=U\left(S_{2}\right)_{i}=1$ for $n i \leq n+m, T$ is a hitting set of $\mathcal{H}$.

Therefore, $p$-NonSeparability*-Test is $\mathrm{W}[2]$-complete, and so $p$-SeParability*-Test is co-W[2]complete.

Lemma 3.6. $\quad p$-SePARABILITY-TEST is co-W[2]-complete.

Proof. Since as proved before in lemma 3.3 that $p$-SeParability*-Test is fpt-reducible to $p$ Separability-Test, and in lemma 3.5 that $p$-Separability*-Test is co-W[2]-complete, $p$-SeparabilityTEST is co-W[2]-hard. Together with lemma 3.2 that $p$-SEParability-TeST $\in$ co-W[2], we obtain that $p$-Separability-Test is co-W[2]-complete.

\section{DISCUSSION}

In this article, we studied the parameterized complexity of three basic problems in pooling design: given an $m \times n$ binary matrix and a positive integer $d$, to decide whether the matrix is $d$-separable $(\bar{d}$-separable, or $d$-disjunct). We proved that these problems are co-W[2]-complete; thus, they do not admit algorithms with running time $f(d) \times(m n)^{O(1)}$ for any computable function $f$. To the best of our knowledge, in general the best known algorithms for the above problems are simply brute-force. It is interesting to investigate whether these problems admit better algorithms.

\section{ACKNOWLEDGMENTS}

Y. Cheng and G. Lin were supported in part by AICML and NSERC. D.-Z. Du was supported in part by the National Science Foundation (grant CCF0621829).

\section{DISCLOSURE STATEMENT}

No competing financial interests exist.

\section{REFERENCES}

Balding, D.J., Bruno, W.J., Knill, E., et al. 1996. A comparative survey of non-adaptive pooling designs, $133-154$. Genetic Mapping and DNA Sequencing. IMA Volumes in Mathematics and Its Applications. Springer-Verlag, New York.

Berger, T., Mandell, J.W., and Subrahmanya, P. 2000. Maximally efficient two-stage group testing. Biometrics 56, 833840.

Chen J., Kanj, I.A., and Jia, W. 2001. Vertex cover: further observations and further improvements. J. Algorithm 41, 280-301. 
Cheng, Y., and Du, D.Z. 2007. Efficient constructions of disjunct matrices with applications to DNA library screening. J. Comput. Biol. 14, 1208-1216.

Cheng, Y., and Du, D.Z. 2008. New constructions of one- and two-stage pooling designs. J. Comput. Biol. 15, $195-205$.

De Bonis, A., Gasieniec, L., and Vaccaro, U. 2005. Optimal two-stage algorithms for group testing problems. $\underline{\text { SIAM }}$ J. Comput. 34, 1253-1270.

Dorfman, R. 1943. The detection of defective members of large populations. Ann. Math. Statist. 14, 436-440.

Downey, R.G., and Fellows, M.R. 1999. Parameterized Complexity. Springer, New York.

Du, D.Z., and Hwang, F.K. 2006. Pooling Designs and Nonadaptive Group Testing: Important Tools for DNA Sequencing. World Scientific, New York.

Du, D.Z., Hwang, F.K., Wu, W., et al. 2006. New construction for transversal design. J. Comput. Biol. 13, 990-995.

Du, D.Z., and Ko, K.I. 1987. Some completeness results on decision trees and group testing, SIAM J. Algebra. Discr. 8 , $762-777$.

D'yachkov, A.G., Macula, A.J., and Rykov, V.V. 2000. New constructions of superimposed codes. IEEE Trans. Inform. Theory 46, 284-290.

D'yachkov, A.G., and Rykov, V.V. 1982. Bounds of the length of disjunct codes. Probl. Contr. Inform. Theory 11, $7-$ 13.

D'yachkov, A.G., Rykov, V.V., and Rashad A.M. 1989. Superimposed distance codes. Probl. Contr. Inform. Theory 18, 237-250.

Eppstein, D., Goodrich, M.T., and Hirschberg, D.S. 2007. Improved combinatorial group testing algorithms for realworld problem sizes. SIAM J. Comput. 36, 1360-1375.

Erdös, P., Frankl, P., and Füredi, Z. 1985. Families of finite sets in which no set is covered by the union of $\mathrm{r}$ others. Israel J. Math. 51, 79-89.

Farach, M., Kannan, S., Knill, E., et al. 1997. Group testing problems with sequences in experimental molecular biology, In B. Carpentieri et al., eds., 357-367. Proceedings of the Compression and Complexity of Sequences. IEEE Press, New York.

Flum, J., and Grohe, M. 2006. Parameterized Complexity Theory. Volume XIV of Texts in Theoretical Computer Science. Springer-Verlag, Berlin.

Fu, H.L., and Hwang F.K. 2006. A novel use of t-packings to construct d-disjunct matrices. Discrete Appl. Math. 154, 1759-1762.

Füredi, Z. 1996. On $r$-cover-free families. J. Combin. Theory Ser. A 73, 172-173.

Hwang, F.K., and Sós, V.T. 1987. Non-adaptive hypergeometric group testing. Studia Sci. Math. Hungar. 22, $257-263$.

Kautz, W.H., and Singleton, R.C. 1964. Nonrandom binary superimposed codes. IEEE Trans. Inform. Theory 10, $363-$ 377.

Knill, E. 1995. Lower bounds for identifying subset members with subset queries. Proc. 6th ACM-SIAM Symp. Discrete Algorithms (SODA' 95) 369-377.

Lichtenstein, O., and Pnueli, A. 1985. Checking that finite state concurrent programs satisfy their linear specification. Proc. 12th ACM Symp. Principles Prog. Lang. (POPL' 85) 97-107.

Macula, A.J. 1996. A simple construction of d-disjunct matrices with certain constant weights. Discrete Math. 162, 311-312.

Macula, A. J. 1999. Probabilistic nonadaptive and two-stage group testing with relatively small pools and DNA library screening. J. Comb. Optim. 2, 385-397.

Ngo, H.Q., and Du, D.Z. 2002. New constructions of non-adaptive and error-tolerance pooling designs. Discrete Math. 243, 161-170.

Olson, M., Hood, L., Cantor, C., et al. 1989. A common language for physical mapping of the human genome. Science 245, 1434-1435.

Park, H., Wu, W., Liu, Z., et al. 2003. DNA screening, pooling design and simplicial complex. J. Comb. Optim. 7, 389394.

Ruszinkó, M. 1994. On the upper bound of the size of the r-cover-free families. J. Combin. Theory Ser. A 66, 302-310.

Address correspondence to:

Dr. Yongxi Cheng

Department of Computing Science

University of Alberta

Edmonton, Alberta T6G 2E8, Canada

E-mail: chengyx@gmail.com 
